EXTENDED REPORT

\title{
Alteration of choroidal circulation in the foveal region in patients with type 2 diabetes
}

\author{
T Nagaoka, N Kitaya, R Sugawara, H Yokota, F Mori, T Hikichi, N Fujio, A Yoshida
}

Br J Ophthalmol 2004;88:1060-1063. doi: 10.1136/bjo.2003.035345

See end of article for authors' affiliations

Correspondence to

T Nagaoka

MD, PhD, Department of

Ophthalmology,

Asahikawa Medical

College, Midorigaoka

Higashi 2-1-1-1,

Asahikawa, 078-8510,

Japan; nagaoka@

asahikawa-med.ac.jp

Accepted for publication 23 December 2003

\begin{abstract}
Aim: To investigate changes in choroidal blood flow (CBF) in the foveal region in patients with type 2 diabetes.

Methods: Laser Doppler flowmetry was used to determine the CBF in the foveal region in 70 patients with type 2 diabetes and 36 age and sex matched healthy subjects (control group). The patients were classified into three groups: 33 patients (33 eyes) with no diabetic retinopathy (NDR), 20 patients (20 eyes) with non-proliferative diabetic retinopathy and no macular oedema (NPDR/MO-), and 17 patients (17 eyes) with NPDR and MO (NPDR/MO+). Optical coherence tomography was also used to measure the foveal thickness.

Results: The group averaged CBF values were 13.5 (4.9), 9.4 (2.5), 10.8 (4.8), and 5.6 (2.0) (arbitrary units) in the control, NDR, NPDR/MO- , and NPDR/MO+ groups, respectively. The group averaged CBF values in the NDR group decreased $(30.2 \% ; p<0.01)$ compared with the control group. The average CBF value in the NPDR/MO+ group was also significantly lower $(48.2 \% ; p<0.01)$ compared with that in the NPDR/MO- group.
\end{abstract}

Conclusion: The CBF in the foveal region significantly decreases in patients with diabetes, especially those with macular oedema.
M uch experimental evidence suggests that retinal blood flow is impaired in patients with diabetes mellitus. ${ }^{1-6}$ However, far less attention has been paid to choroidal blood flow (CBF). Some investigators measured the change in pulsatile ocular blood flow (POBF) in patients with diabetes by studying the change in $\mathrm{CBF}$, and they reported decreased POBF in patients with no diabetic retinopathy $(\mathrm{NDR})^{7}$ and background diabetic retinopathy. ${ }^{8}$ In contrast, others have reported that the POBF increased ${ }^{9}$ or was unchanged $^{10}$ in all grades of diabetic retinopathy. Thus, the changes in $\mathrm{CBF}$ in patients with diabetes remain controversial.

Diabetic macular oedema is the most common cause of visual impairment and legal blindness in patients with type 2 diabetes, ${ }^{11} 12$ but the pathogenesis of diabetic macular oedema is still poorly understood. Because the choroidal vasculature, especially the choriocapillaris, provides oxygen and nutrients to the outer retina and is presumed to be responsible for maintaining the highly metabolically active photoreceptor cells, ${ }^{13}$ impairment of the choriocapillaris may cause severe functional damage to the retina in the foveal region, which has no retinal vasculature.

In this study, we hypothesised that impaired CBF in the foveal region has a role in the pathogenesis of diabetic retinopathy and macular oedema. Riva et al ${ }^{14}$ used laser Doppler flowmetry to assess the CBF in the human foveal region and reported that the measurements obtained corresponded primarily to determinations of choriocapillary flow. To our knowledge, no study has evaluated the CBF in the foveal region in patients with diabetes using this technique. Therefore, we assessed the foveal choroidal circulation in patients with diabetes using laser Doppler flowmetry and compared the results with those of age matched healthy subjects.

\section{SUBJECTS AND METHODS Study design}

Seventy eyes of 70 patients with type 2 diabetes and 36 eyes of 36 age and sex matched healthy volunteers (control group) were included in this study. All procedures performed in the study followed the tenets of the Declaration of Helsinki. After a detailed explanation of the procedures, written informed consent was obtained from all participants.

Each subject underwent a complete ophthalmological examination before the blood flow was measured. All had adequate pupillary dilation and clear media. The patients with diabetes were classified into three groups: 33 patients (33 eyes) with NDR, 20 patients (20 eyes) with nonproliferative diabetic retinopathy and no macular oedema (NPDR/MO-), and 17 patients (17 eyes) with NPDR and macular oedema (NPDR/MO+). Macular oedema was evaluated by slit lamp biomicroscopy with a preset lens as described previously. ${ }^{15}$ Briefly, clinically significant macular oedema was graded based on the presence or absence of macular thickening or hard exudates at or within $500 \mu \mathrm{m}$ of the central macula, as defined by the Early Treatment Diabetic Retinopathy Study protocol. ${ }^{16}{ }^{17}$ Exclusion criteria included previous ocular surgery, a history of macular photocoagulation, proliferative diabetic retinopathy, rubeosis iridis, epiretinal membrane, macular degeneration, poor quality optic coherent tomography (OCT) scans due to cataract, visual acuity of 20/40 or less, and high ametropia (spherical equivalent worse than -5.0 dioptres (D) or greater than +3.0 D). Patients with hypertension (>160/95 mm Hg) and other systemic complications were also excluded from the study.

\section{Study protocol}

Blood flow measurements were obtained from one randomly chosen eye of each participant. Patients with hard exudates or retinal pigment epithelial degeneration within $500 \mu \mathrm{m}$ of

Abbreviations: $\mathrm{CBF}$, choroidal blood flow; $\mathrm{CBVel}$, foveolar choroidal blood velocity; $\mathrm{CBV}$ ol, foveolar blood volume; NDR, no diabetic retinopathy; POBF, pulsatile ocular blood flow; NPDR/MO-, diabetic retinopathy and no macular oedema; NPDR/MO+, diabetic retinopathy and macular oedema; OCT, optical coherence tomography; VEGF, vascular endothelial growth factor 
the central macula were excluded to prevent blockage of the laser beam, which could affect the sample volume and alter the scattering of the laser light.

A rest period of at least 20 minutes was scheduled for each subject. The temperature in the examination room was maintained from $22^{\circ} \mathrm{C}$ to $24^{\circ} \mathrm{C}$. The subjects were asked to abstain from drinking coffee and smoking for at least 2 hours before the test.

The systolic, diastolic, mean arterial blood pressure, and heart rate were estimated by electronic sphygmomanometer (EP-88Si, Colin, Tokyo, Japan). Intraocular pressure (IOP) was monitored by applanation tonometry (Haag Streit, Bern, Switzerland). Pupil dilation was achieved using a combination of $0.5 \%$ tropicamide and $1 \%$ phenylephrine eye drops.

\section{Measurement of CBF in the foveal region}

Determinations of relative foveolar choroidal blood velocity (CBVel), foveolar choroidal blood volume (CBVol), and CBF were obtained using a method based on the laser Doppler velocity flowmetry technique, ${ }^{18}$ the details of which have been published previously. ${ }^{14}$ Briefly, a diode laser $(670 \mathrm{~nm}$ ) with an intensity of $20 \mu \mathrm{W}$ was delivered through a fundus camera (Model TRC; Topcon, Tokyo, Japan). The diameter of the probing laser beam was approximately $200 \mu \mathrm{m}$. The velocity is expressed in hertz and volume and flux were expressed in arbitrary units. In our previous study, the average coefficients of variability of CBVol, CBVel, and CBF in healthy normal subjects $(\mathrm{n}=5)$ were $12.7 \%, 10.0 \%$, and $6.8 \%$, respectively, ${ }^{19}$ suggesting that we could measure these parameters with good reproducibility.

Subjects fixated on the probing laser beam to determine the foveolar CBF. During blood flow measurements, proper fixation was ascertained by direct observations of the foveola through the fundus camera. All measurements were performed with the subjects seated in a dark room.

The choroidal circulation was measured continuously in each participant for about 30 seconds. The measurements were performed twice in each subject. All these flow parameters then were averaged over two periods of 30 seconds. Before the data were analysed, spikes caused by micromovements and blinks were removed as described previously. ${ }^{20}$ Data analysis was performed by a masked observer using a computer (NeXT Computer, Redwood City, CA, USA) with software specifically developed to analyse Doppler signals from ocular tissues. ${ }^{21}$

\section{Retinal thickness measurements}

OCT (Zeiss-Humphrey Inc., Dublin, CA, USA, with software application version A4.1), a technique for high resolution cross sectional retinal imaging, was used to measure the retinal thickness obtained in a radial spoke pattern centred on the fovea. ${ }^{16}$ The principles of OCT have been described previously. ${ }^{22-24}$ Briefly, six radiating $5 \mathrm{~mm}$ OCT scans at intervals of $30^{\circ}$ were obtained. The images were automatically analysed by the OCT computer software using the "retina map" algorithm, which determines retinal thickness from the
OCT section by an edge detection technique that uses the reflection pattern from the cross section.

\section{Statistical analysis}

All values are expressed as the mean (SD). The assumption of normality of the data was assessed using the Shapiro-Wilk test. For statistical analysis, we used one way analysis of variance (ANOVA) followed by post hoc comparison with Dunnett's procedure. A value of $\mathrm{p}<0.05$ was considered statistically significant.

\section{RESULTS}

No significant differences in age, mean arterial blood pressure, heart rate, or IOP were observed among the groups (table 1). In patients with diabetes, there were no significant differences in the duration of diabetes mellitus, or haemoglobin $A_{1 c}$ value, and fasting blood sugar were observed among the three diabetic groups (table 1).

The group averaged values of the CBVol, CBVel, CBF, and foveal thickness are shown in table 2 . There were substantial differences in the CBVel between the controls and all diabetic groups. However, a significant decrease in the CBVol was observed only in the NPDR/MO+ group compared with the controls. There were significant differences in CBF between the controls and the three diabetic groups $(p<0.01)$. In patients with diabetes but with NDR, the average CBF was $30.2 \%$ lower than that in the controls $(\mathrm{p}<0.01)$. In addition, there were also significant differences in CBF between both the NDR and the NPDR/MO - and the NPDR/MO+ groups $(p<0.01)$. The average CBF in the NPDR/MO+ group was $48.2 \%$ lower than that in the NPDR/MO- group and was $58.5 \%$ lower than in the control group. Although the CBVel was significantly lower in all three diabetic groups compared with the control group, the CBVol was significantly reduced compared with the control group only in the NPDR/MO+ group. Therefore, the observed decreases in CBF in the NDR and NPDR/MO - groups seem to be associated with decreased CBVel. The evaluation of foveal thickness by OCT provided us with the expected data. The average foveal thickness values in the NPDR/MO+ group were significantly higher than in the other three groups $(\mathrm{p}<0.01$, table 2$)$.

\section{DISCUSSION}

In the present study, we quantified the change in the CBF in the foveal region in patients with diabetes using laser Doppler flowmetry. In addition, the group averaged CBF value in the NPDR/MO+ group was lower than that in the NPDR/MOgroup. These results indicate that the $\mathrm{CBF}$ in the foveal region may decrease in the early stage of diabetic retinopathy and decrease further in the presence of macular oedema.

Because the measurements obtained by laser Doppler flowmetry correspond primarily to determinations of choriocapillary flow, ${ }^{14}$ our findings that the CBF values obtained in the NDR group were lower than that in the age matched control group (table 2 ) suggest that the circulatory changes in

Table 1 Characteristics of control subjects and subjects with diabetic mellitus

\begin{tabular}{lllll}
\hline & Control & NDR & NPDR/MO - & NPDR/MO+ \\
\hline Number (male/female) & $36(18 / 18)$ & $33(14 / 19)$ & $20(9 / 11)$ & $17(9 / 8)$ \\
Age (years) & $59.8(13.0)$ & $62.2(12.1)$ & $61.3(14.2)$ & $63.6(11.4)$ \\
MABP (mm Hg) & $98.6(14.7)$ & $96.1(16.1)$ & $94.8(12.5)$ & $98.7(14.6)$ \\
IOP (mm Hg) & $14.1(3.3)$ & $14.4(2.2)$ & $15.0(4.0)$ & $14.2(3.0)$ \\
HR (beats/min) & $70.7(9.6)$ & $71.4(10.9)$ & $72.9(12.0)$ & $72.1(10.8)$ \\
HbA 1 (\%) & - & $7.4(1.5)$ & $8.4(1.6)$ & $8.6(2.0)$ \\
FBS (g/dl) & - & $189.3(72.7)$ & $161.7(57.9)$ & $200.9(49.4)$ \\
\hline
\end{tabular}

$M A B P=$ mean arterial blood pressure; $I O P=$ intraocular pressure; $H R=$ heart rate; $F B S=$ fasting blood sugar.

Values are expressed as means (SD). 
Table 2 The group averaged values of choroidal circulation and foveal thickness in each group

\begin{tabular}{lllll}
\hline & Control & NDR & NPDR/MO- & NPDR/MO+ \\
\hline CBVel (Hz) & $353.8(80.6)$ & $323.4(81.4)^{*}$ & $298.8(67.8)^{*}$ & $279.1(90.4) \dagger$ \\
CBVol (au) & $0.63(0.21)$ & $0.58(0.30)$ & $0.63(0.33)$ & $0.50(0.32)^{*}$ \\
CBF (au) & $13.5(4.9)$ & $9.4(2.5) \dagger$ & $10.8(4.8)^{*}$ & $5.6(2.0) \dagger$ \\
foveal thickness $(\mu \mathrm{m})$ & $173.2(28.2)$ & $180.1(29.9)$ & $188.3(34.8)$ & $275.4(107.5) \dagger$ \\
\hline
\end{tabular}

$M A B P=$ mean arterial blood pressure; $I O P=$ intraocular pressure; $H R=$ heart rate; $F B S=$ fasting blood sugar.

Values are expressed as means (SD).

${ }^{*} p<0.05$ and $+p<0.01$ compared with control.

$p<0.05$ and $p<0.01$ compared with the NPDR/MO- group.

CBVel indicates choroidal blood velocity; CBVol choroidal blood volume; and CBF choroidal blood flow.

the choriocapillaris may occur before the clinical manifestations of diabetic retinopathy. Previous histopathological studies demonstrated the dropout of the choriocapillaris in diabetic eyes. ${ }^{25-27}$ The dropout of the choriocapillaris could increase vascular resistance, resulting in decreased blood flow in the choriocapillaris. Therefore, it is likely that the changes in circulation in the choriocapillaris may agree with those histological findings.

The abnormalities of ocular blood flow in patients with diabetes have been examined in many studies. The CBF was reported to decrease, increase, and remain unchanged in patients with diabetes in POBF and colour Doppler imaging. ${ }^{8-10}$ In our recent study, the POBF did not change in any stage in diabetes, ${ }^{28}$ suggesting that the blood flow in the large choroidal vessel, which POBF mainly reflect, may not be affected in patients with diabetes. Although we did not measure the POBF and perform laser Doppler flowmetry simultaneously in patients with diabetes, it is likely that the mechanisms of the impairment of choroidal circulation may not be similar in large choroidal vessels and the choriocapillaris, especially in the foveal lesion.

This study had limitations, especially concerning the methodology used to measure the CBF. Although the values of laser Doppler flowmetry obtained from the patients with age related macular degeneration ${ }^{29}$ and glaucoma ${ }^{30}$ were compared with age matched healthy subjects in previous studies, comparisons of relative blood flow measurements between different group of individuals, such as normal subjects and patients with diabetes, must be done with care because the assessment of blood flow depends on the optical and scattering properties of the tissues. ${ }^{14}$ It is at least reasonable to consider that the decreased $\mathrm{CBF}$ in the foveal region in the NDR group is a disease related alteration, because there was no difference in the foveal thickness between the healthy subjects and the patients with diabetes with no retinopathy.

In the patients with NPDR and diabetic macular oedema, both blood volume and blood velocity were significantly lower than in NPDR/MO - group (table 2), which suggests that the $\mathrm{CBF}$ in the foveal region may decrease more in patients with diabetes with macular oedema. On the other hand, another possible explanation for the decreased CBF in the NPDR/MO+ group should be considered. The present data do not provide a definitive explanation for this, because it is possible that the increased retinal thickness may affect the sampled volume and alter the scattering behaviour of the laser light, resulting in the decreased intensity of the Doppler shift. If the increase in sampling volume by macular oedema mainly affected the present data obtained using a laser Doppler flowmetry technique, any negative correlation between the foveal thickness and CBF should be observed. However, there was no such a correlation in NPDR/MO+ group (data not shown). We therefore speculate that the present data may be a result of disease related alterations in $\mathrm{CBF}$, which are greater than those produced by the change in the sampling volume due to macular oedema. A follow up study is needed to investigate whether the decreased CBF in the foveal region causes macular oedema in patients with NPDR with no diabetic macular oedema.

Our results may provide a new clue to the mechanism of diabetic macular oedema. It is likely that the decreased blood flow in the choriocapillaris that we observed may cause severe retinal tissue hypoxia because the choriocapillaris is the major source of nutrition for the retinal pigment epithelium and outer retinal layers in the foveal region. In the presence of tissue hypoxia, the expression of vascular endothelial growth factor (VEGF), which was found to increase vascular permeability, was reported to increase in retinal cells (retinal pigment epithelial, pericytes, and microvascular endothelial cells in vitro). ${ }^{31}$ Previous studies also indicated that VEGF induces breakdown of the bloodretinal barrier, which is an important mechanism of diabetic macular oedema, ${ }^{32}$ in patients with diabetes ${ }^{34}$ and in diabetic rats. ${ }^{35}$ Indeed, the level of VEGF was recently reported to be elevated in the vitreous fluid of patients with severe diabetic macular oedema. ${ }^{36}$ Taken together, we interpret these results to indicate that the decreased CBF in the foveal region that we observed may cause tissue hypoxia and consequently increase the level of VEGF in the foveal region of the retina, resulting in the development of macular oedema as a result of breakdown of the blood-retinal barrier.

Because this was a cross sectional study, we cannot reach a definitive conclusion about whether the decreased blood flow is a primary factor in the development of diabetic macular oedema or is the result of the loss of cellular components that occurs secondarily in diabetic macular oedema. Further prospective studies are necessary to determine the precise role of changes in $\mathrm{CBF}$ in the development of diabetic macular oedema.

In conclusion, our results showed that the CBF in the foveal region significantly decreases in patients with diabetes, especially those with macular oedema. Moreover, the decrease in $\mathrm{CBF}$ in the foveal region may be associated with the development of diabetic macular oedema.

\section{ACKNOWLEDGEMENT}

This work was supported in part by grant in aid for young scientists (B) 14770940 (TN), 15790970 (FM), and 14571652 (AY).

\section{Authors' affiliations}

T Nagaoka, N Kitaya, R Sugawara, H Yokota, F Mori, T Hikichi, N Fujio, A Yoshida, Department of Ophthalmology, Asahikawa Medical College, Asahikawa, Japan

\section{REFERENCES}

1 Bursell SE, Clermont AC, Kinsley BT, et al. Retinal blood flow changes in patients with insulin-dependent diabetes mellitus and no diabetic retinopathy. Invest Ophthalmol Vis Sci 1996;37:886-97.

2 Clermont AC, Aiello LP, Mori F, et al. Vascular endothelial growth factor and severity of nonproliferative diabetic retinopathy mediate retinal hemodynamics in vivo: a potential role for vascular endothelial growth factor in the progression of nonproliferative diabetic retinopathy [see comments]. Am J Ophthalmol 1997; 124:433-46. 
3 Konno S, Feke GT, Yoshida A, et al. Retinal blood flow changes in type I diabetes. A long-term follow-up study. Invest Ophthalmol Vis Sci 1996;37:1140-8.

4 Patel V, Rassam S, Newsom R, et al. Retinal blood flow in diabetic retinopathy. BMJ 1992;305:678-83.

5 Yoshida A, Feke GT, Morales-Stoppello J, et al. Retinal blood flow alterations during progression of diabetic retinopathy. Arch Ophthalmol 1983;101:225-7.

6 Grunwald JE, Riva CE, Baine J, et al. Total retinal volumetric blood flow rate in diabetic patients with poor glycemic control. Invest Ophthalmol Vis Sci 1992;33:356-63.

7 Geyer O, Neudorfer M, Snir T, et al. Pulsatile ocular blood flow in diabetic retinopathy. Acta Ophthalmol Scand 1999;77:522-5.

8 Langham ME, Grebe R, Hopkins S, et al. Choroidal blood flow in diabetic retinopathy. Exp Eye Res 1991;52:167-73.

9 MacKinnon JR, O'Brien C, Swa K, et al. Pulsatile ocular blood flow in untreated diabetic retinopathy. Acta Ophthalmol Scand 1997;75:661-4.

10 Schmidt KG, von Ruckmann A, Kemkes-Matthes B, et al. Ocular pulse amplitude in diabetes mellitus. Br J Ophthalmol 2000;84:1282-4.

11 Klein R, Moss SE, Klein BE, et al. The Wisconsin epidemiologic study of diabetic retinopathy. XI The incidence of macular edema. Ophthalmology 1989;96:1501-10.

12 Moss SE, Klein R, Klein BE. The 14-year incidence of visual loss in a diabetic population. Ophthalmology 1998;105:998-1003.

13 Alm A, Bill A. Ocular circulation. Adler's physiology of the eye. 9th ed. St Lovis: Mosby, 1992:198-227.

14 Riva CE, Cranstoun SD, Grunwald JE, et al. Choroidal blood flow in the foveal region of the human ocular fundus. Invest Ophthalmol Vis Sci 1994; $35: 4273-8$

15 Hikichi T, Fujio N, Akiba J, et al. Association between the short-term natural history of diabetic macular edema and the vitreomacular relationship in type II diabetes mellitus. Ophthalmology 1997;104:473-8.

16 Hee MR, Puliafito CA, Duker JS, et al. Topography of diabetic macular edema with optical coherence tomography. Ophthalmology 1998;105:360-70.

17 ETDRS. Early Treatment Diabetic Retinopathy Study design and baseline patient characteristics. ETDRS report number 7. Ophthalmology 1991;98:741-56.

18 Riva CE, Cranstoun SD, Mann RM, et al. Local choroidal blood flow in the cat by laser Doppler flowmetry. Invest Ophthalmol Vis Sci 1994;35:608-18.

19 Yoshida A. New examination methods for macular disorders-application of diagnosis and treatment. Nippon Ganka Gakkai Zasshi 2000;104:899-942.

20 Movaffaghy A, Chamot SR, Petrig BL, et al. Blood flow in the human optic nerve head during isometric exercise. Exp Eye Res 1998;67:561-8.
21 Petrig BL, Riva CE. Optic nerve head laser Doppler flowmetry: Principles and computer analysis. In: Kaiser HJ, Flammer J, Hendrickson PH, eds. Ocular blood flow. Basel: Kaeger, 1996:120-7.

22 Hee MR, Puliafito CA, Wong C, et al. Optical coherence tomography of macular holes. Ophthalmology 1995;102:748-56.

23 Puliafito CA, Hee MR, Lin CP, et al. Imaging of macular diseases with optical coherence tomography. Ophthalmology 1995;102:217-29.

24 Polito A, Shah SM, Haller JA, et al. Comparison between retinal thickness analyzer and optical coherence tomography for assessment of foveal thickness in eyes with macular disease. Am J Ophthalmol 2002;134:240-51

25 Hidayat AA, Fine BS. Diabetic choroidopathy. Light and electron microscopic observations of seven cases. Ophthalmology 1985;92:512-22.

26 McLeod DS, Lutty GA. High-resolution histologic analysis of the human choroidal vasculature. Invest Ophthalmol Vis Sci 1994;35:3799-811.

27 Lutty GA, Cao J, McLeod DS. Relationship of polymorphonuclear leukocytes to capillary dropout in the human diabetic choroid. Am J Pathol 1997;151:707-14

28 Mori F, Yokota H, Nagaoka T, et al. Pulsatile ocular blood flow study: unaffected in type 2 diabetic mellitus. Jpn J Ophthalmol 2003;47:621-2.

29 Grunwald JE, Hariprasad SM, DuPont J, et al. Foveolar choroidal blood flow in age-related macular degeneration. Invest Ophthalmol Vis Sci 1998;39:385-90

30 Grunwald JE, Piltz J, Hariprasad SM, et al. Optic nerve and choroidal circulation in glaucoma. Invest Ophthalmol Vis Sci 1998;39:2329-36.

31 Aiello LP, Northrup JM, Keyt BA, et al. Hypoxic regulation of vascular endothelial growth factor in retinal cells. Arch Ophthalmol 1995; 113:1538-44.

32 Cunha-Vaz JG, Goldberg MF, Vygantas C, et al. Early detection of retinal involvement in diabetes by vitreous fluorophotometry. Ophthalmology 1979;86:264-75

33 Mori F, Hikichi T, Takahashi J, et al. Dysfunction of active transport of bloodretinal barrier in patients with clinically significant macular edema in type 2 diabetes. Diabetes Care 2002;25:1248-9.

34 Vinores SA, Youssri Al, Luna JD, et al. Upregulation of vascular endothelial growth factor in ischemic and non ischemic human and experimental retinal disease. Histol Histopathol 1997; 12:99-109.

35 Qaum $T, X \cup Q$, Joussen $A M$, et al. VEGF-initiated blood-retinal barrier breakdown in early diabetes. Invest Ophthalmol Vis Sci 2001;42:2408-13.

36 Funatsu H, Yamashita H, lkeda T, et al. Angiotensin II and vascular endothelial growth factor in the vitreous fluid of patients with diabetic macular edema and other retinal disorders. Am J Ophthalmol 2002;133:537-43. 